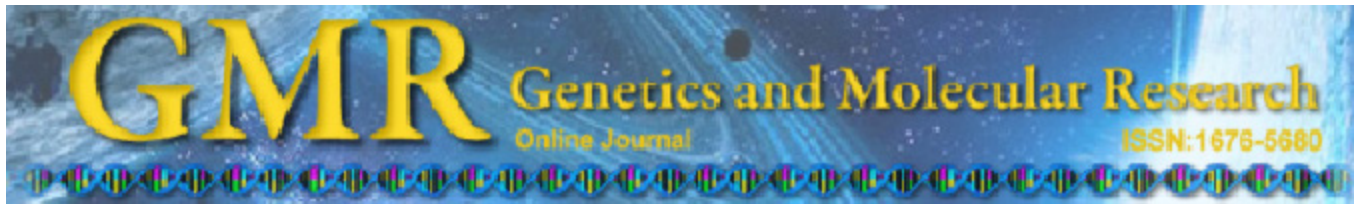

\title{
Association of serum adipose triglyceride lipase levels with obesity and diabetes
}

\author{
L. Yang ${ }^{1 *}$, S.J. Chen ${ }^{1 *}$, G.Y. Yuan ${ }^{1}$, L.B. Zhou ${ }^{2}$, D. Wang ${ }^{1}$, X.Z. Wang ${ }^{1}$ \\ and J.J. Chen ${ }^{1}$ \\ ${ }^{1}$ Department of Endocrinology, Affiliated Hospital of Jiangsu University, \\ Zhenjiang, Jiangsu Province, China \\ ${ }^{2}$ Ruijin Hospital Affiliated Medical School, \\ Shanghai Institute of Endocrine and Metabolic Diseases, \\ Shanghai Jiaotong University, Shanghai, China \\ *These authors contributed equally to this study. \\ Corresponding author: G.Y. Yuan \\ E-mail: yuanguoyue@yeah.net / yuanguoyue@hotmail.com
}

Genet. Mol. Res. 13 (3): 6746-6751 (2014)

Received May 27, 2013

Accepted April 28, 2014

Published August 28, 2014

DOI http://dx.doi.org/10.4238/2014.August.28.18

\begin{abstract}
The aim of this study was to detect the serum adipose triglyceride lipase (ATGL) levels in obesity and newly diagnosed type 2 diabetes patients, and to explore the association between ATGL with glucose and lipid metabolism. We enrolled 66 patients with type 2 diabetes and 48 patients with normal glucose regulation, who were divided into an overweight or obese subgroup and a normal weight subgroup according to body mass index $(\mathrm{BMI}) \geq 25 \mathrm{~kg} / \mathrm{m}^{2}$. The enzyme-linked immunosorbent assay was used to detect fasting blood glucose, blood lipids, fasting insulin, and ATGL levels. The serum ATGL level in the overweight or obese group was lower than that in the non-obese group including patients with type 2 diabetes and normal glucose regulation: $239 \pm 61$ vs $355 \pm 54$ $\mu \mathrm{g} / \mathrm{L}$ and $242 \pm 60 v s 383 \pm 58 \mu \mathrm{g} / \mathrm{L}$, respectively $(t=22.53, t=8.23, \mathrm{P}<$ $0.05)$. The Pearson correlation analysis showed that fasting serum ATGL was negatively correlated with body fat content, BMI, waist-to-hip ratio,
\end{abstract}


triglycerides, and the homeostatic model assessment-insulin resistance level $(r=-0.271, r=-0.238, r=-0.375, r=-0.313$, and $r=-0.164$, respectively, $\mathrm{P}<0.05)$. The stepwise regression analysis showed that the waist-to-hip ratio and body fat content were independently associated with the serum ATGL level. Our results indicated that the ATGL level may be closely related to obesity.

Key words: Diabetes; Obesity; Lipase

\section{INTRODUCTION}

A large number of studies have found that fat is not only an energy storage organ, but is also an endocrine organ that is involved in regulating endocrine and inflammatory responses. Abnormalities in the lipid metabolism of fat cells lead to adipose tissue dysfunction, and are closely related to the occurrence and development of many diseases. The newly discovered adipose triglyceride lipase (ATGL) was shown to be the rate-limiting enzyme of lipid hydrolysis (Zimmermann et al., 2004), and was found to play an important role in the regulation of energy metabolism. This study aimed to investigate the correlation between serum ATGL levels and obesity and diabetes.

\section{MATERIAL AND METHODS}

\section{Subjects}

A total of 114 patients, including 54 males and 60 females aged 38 to 70 years, were selected from August 2006 to April 2009 in the clinical department and the endocrinology department of our hospital. According to the World Health Organization (WHO) diabetes diagnostic criteria (1999) and the WHO obesity diagnostic criteria (1998), 48 patients were enrolled in the normal glucose regulation group and 66 patients were enrolled in the newly diagnosed type 2 diabetes mellitus (DM) group. The subjects were then further divided into the overweight or obese subgroup and the normal weight subgroup based on whether the body mass index $(\mathrm{BMI}) \geq 25 \mathrm{~kg} / \mathrm{m}^{2}$. The normal weight and normal glucose regulation group (NW-NGR) contained 21 patients. The overweight or obese and normal glucose regulation group (OW/OB-NGR) contained 27 patients. The normal weight and type 2 diabetes group (NW-T2DM) contained 36 patients. The overweight or obese and type 2 diabetes group (OW/ OB-T2DM) contained 30 patients. All DM patients were newly diagnosed and did not receive any treatment prior to the study, including diet, exercise, and medication. Type 1 DM patients, female patients at childbearing age, such as contraceptive drug takers, secondary obesity, and DM patients caused by Cushing's syndrome, were excluded from the study. All included patients had no history of ketoacidosis, coronary heart disease, kidney disease, or other serious complications of DM. Patients with hypertension controlled their blood pressure below $140 / 90 \mathrm{mmHg}(1 \mathrm{mmHg}=0.133 \mathrm{kPa})$ with hypertensive medication.

\section{Determination of anthropometric parameters}

Height and weight of patients were measured without shoes or hats and only in un- 
derwear. The same conditions were applied to all patients, and the accuracies of height $(\mathrm{cm})$ and weight $(\mathrm{kg})$ were $0.1 \mathrm{~kg}$ and $0.1 \mathrm{~cm}$, respectively. BMI was calculated as: weight $/$ height $^{2}$ $\left(\mathrm{kg} / \mathrm{m}^{2}\right)$. According to the methods recommended by the WHO (1995), waist circumference was measured as the horizontal circumference at the midpoint of the lower edge of the ribs and the iliac ridge line. Hip circumference was measured as the maximum circumference of the buttocks. The accuracy of circumference measurements was $0.1 \mathrm{~cm}$. The waist-to-hip ratio (WHR) was calculated as: waist/hip and were compared to the normal ratios of $<0.90$ in males and $<0.85$ in females. Body fat percentage was calculated as: $1.2 \times$ BMI $+0.23 \times$ age -16.2 in males, and body fat $(\%)=1.2 \mathrm{x}$ BMI $+0.23 \mathrm{x}$ age -5.4 in females (Deurenberg et al., 1991).

\section{Specimen collection and processing}

The venous blood of patients fasted for 12-14 $\mathrm{h}$ was taken in the morning. Blood glucose, plasma total cholesterol (TC), triglyceride (TG), high-density lipoprotein cholesterol (HDL-C), low-density lipoprotein cholesterol (LDL-C), and blood insulin levels were measured. Some serum was separated and stored at $-20^{\circ} \mathrm{C}$ for further use. The Human ATGL enzyme-linked immunosorbent assay (ELISA) kit was purchased from Phoenix Pharmaceuticals (USA); the lowest measurable concentration was $0.05 \mu \mathrm{g} / \mathrm{L}$ with the inter-batch $\mathrm{CV}<$ $6 \%$ and the intra-batch $\mathrm{CV}<4 \%$. The homeostatic model assessment-insulin resistance index (HOMA-IR) was calculated with the following formula: HOMA-IR $=$ [fasting glucose $(\mathrm{mM})$ $\mathrm{x}$ fasting insulin $(\mathrm{mU} / \mathrm{L})] / 22.5$.

\section{Statistical analysis}

The SPSS10.0 statistical software was used. The Student $t$-test, the $\chi^{2}$ test, linear correlation analysis, and stepwise multiple regression were used according to research purposes and data types. Data are reported as means \pm standard error.

\section{RESULTS}

As shown in Table 1, there was no difference in age or gender among the groups. The body fat parameters (BMI, body fat content, waist circumference, and WHR) in the obese group (including overweight or obese patients with normal glucose regulation and type 2 diabetes) were significantly higher than those in the non-obese group (including normal weight patients with normal glucose regulation and type 2 diabetes), and the difference was statistically significant $(\mathrm{P}<0.05)$. Among the obese patients, there was no significant difference in body fat parameters (BMI, body fat content, waist circumference, and WHR) between the OW/OB-NGR group and the OW/OB-T2DM group $(t=0.83, t=0.48, t=0.47, t=0.06$, respectively; $\mathrm{P}>0.05$ ). There was also no significant difference in body fat parameters, including BMI and body fat content, among the non-obese patients between the NW-NGR group and the NW-T2DM group $(t=1.22$ and $t=1.36$, respectively; P $>0.05)$. However, the WHR and waist circumference were significantly increased in the NW-T2DM group, and the difference was statistically significant $(t=3.32$ and $t=2.58 ; \mathrm{P}<0.05)$. The fasting blood glucose level in the type 2 diabetes group was significantly higher than that in the normal glucose regulation group, and the difference was statistically significant $(t=26.32, \mathrm{P}<0.05)$. The fasting insulin was the highest in the OW/OB-NGR group. The HOMA-IR values in the NW-T2DM group, 
OW/OB-NGR group, and NW-NGR group against the contrast group were significantly different $(t=19.04, t=19.55, t=13.87, \mathrm{P}<0.05)$.

\begin{tabular}{|c|c|c|c|c|c|c|c|}
\hline Group & $\begin{array}{c}\text { Case } \\
\text { (Male/Female) }\end{array}$ & $\begin{array}{l}\text { Age } \\
\text { (years) }\end{array}$ & $\begin{array}{l}\text { Waist circumference } \\
(\mathrm{cm})\end{array}$ & $\begin{array}{c}\mathrm{BMI} \\
\left(\mathrm{kg} / \mathrm{m}^{2}\right)\end{array}$ & $\begin{array}{l}\text { Waist-to- } \\
\text { hip ratio }\end{array}$ & $\begin{array}{l}\text { Body fat } \\
\text { content }\end{array}$ & HOMA-IR \\
\hline \multicolumn{8}{|l|}{ NGR group } \\
\hline NW-NGR & $21(10 / 11)$ & $57 \pm 6$ & $79 \pm 5$ & $21 \pm 3$ & $0.83 \pm 0.04$ & $29 \pm 7$ & $1.6 \pm 0.7$ \\
\hline OW/OB-NGR & $27(12 / 15)$ & $59 \pm 11$ & $93 \pm 10^{\mathrm{a}}$ & $28 \pm 4^{\mathrm{a}}$ & $0.91 \pm 0.08^{\mathrm{a}}$ & $33 \pm 6^{a}$ & $4.0 \pm 0.5^{\mathrm{a}}$ \\
\hline \multicolumn{8}{|l|}{ T2DM group } \\
\hline NW-T2DM & $36(18 / 18)$ & $58 \pm 12$ & $83 \pm 6^{b}$ & $23 \pm 3^{b}$ & $0.86 \pm 0.13^{\mathrm{ab}}$ & $28 \pm 6^{\mathrm{b}}$ & $4.2 \pm 0.3^{\mathrm{a}}$ \\
\hline OW/OB-T2DM & $30(14 / 16)$ & $56 \pm 7$ & $94 \pm 5^{\mathrm{ac}}$ & $27 \pm 5^{\mathrm{ac}}$ & $0.94 \pm 0.07^{\mathrm{ac}}$ & $32 \pm 9^{c}$ & $4.8 \pm 0.5^{\mathrm{a}}$ \\
\hline Group & $\begin{array}{c}\mathrm{TC} \\
(\mathrm{mM})\end{array}$ & $\begin{array}{l}\text { Triglycerides } \\
\text { (mM) }\end{array}$ & $\begin{array}{l}\text { HDL-C } \\
(\mathrm{mM})\end{array}$ & $\begin{array}{c}\text { LDL-C } \\
(\mathrm{mM})\end{array}$ & $\begin{array}{l}\text { Fasting glucose } \\
(\mathrm{mM})\end{array}$ & $\begin{array}{c}\text { FINS } \\
(\mathrm{mU} / \mathrm{L})\end{array}$ & $\begin{array}{l}\text { ATGL } \\
(\mathrm{ng} / \mathrm{mL})\end{array}$ \\
\hline \multicolumn{8}{|l|}{ NGR group } \\
\hline NW-NGR & $4.2 \pm 1.1$ & $1.1 \pm 0.3$ & $1.63 \pm 0.29$ & $2.1 \pm 0.6$ & $5.2 \pm 4.0$ & $9 \pm 3$ & $383 \pm 58$ \\
\hline OW/OB-NGR & $4.6 \pm 0.7$ & $1.3 \pm 0.9$ & $1.47 \pm 0.51$ & $2.4 \pm 0.6$ & $5.2 \pm 2.9$ & $20 \pm 6^{\mathrm{a}}$ & $242 \pm 60^{\mathrm{a}}$ \\
\hline \multicolumn{8}{|l|}{ T2DM group } \\
\hline NW-T2DM & $5.2 \pm 1.0$ & $1.7 \pm 1.2$ & $1.31 \pm 0.41$ & $3.0 \pm 0.7$ & $10.9 \pm 3.4^{\mathrm{ab}}$ & $15 \pm 8^{a}$ & $355 \pm 54^{\mathrm{b}}$ \\
\hline OW/OB-T2DM & $5.4 \pm 2.4$ & $3.2 \pm 1.4^{\mathrm{abc}}$ & $1.20 \pm 0.68^{\mathrm{abc}}$ & $3.2 \pm 1.9$ & $9.6 \pm 3.4 \mathrm{a}^{\mathrm{b}}$ & $18 \pm 9^{\mathrm{a}}$ & $239 \pm 61^{\text {ac }}$ \\
\hline
\end{tabular}

Data are reported as means \pm SE. NGR $=$ normal glucose regulation; T2DM $=$ type 2 Diabetes; NW-NGR $=$ normal glucose regulation-normal weight; OW/OB-NGR = normal glucose regulation-overweight or obese; NW-T2DM = type 2 diabetes-normal weight; OW/OB-T2DM = type 2 diabetes-overweight or obese; HOMA-IR = insulin resistance index; TC $=$ total cholesterol; HDL-C = high-density lipoprotein cholesterol; LDL-C = low-density lipoprotein cholesterol; FINS $=$ fasting insulin; ATGL = adipose triglyceride lipase. ${ }^{\text {a }} \mathrm{P}<0.05$ compared with the NW-NGR group. ${ }^{b} \mathrm{P}<0.05$ compared with OW/OB-NGR group. ${ }^{c} \mathrm{P}<0.05$ compared with the NW-T2DM group.

The serum ATGL levels in the four groups were as follows: $383 \pm 58 \mu \mathrm{g} / \mathrm{L}$ in the NW-NGR group, $355 \pm 54 \mu \mathrm{g} / \mathrm{L}$ in the NW-T2DM group, $242 \pm 60 \mu \mathrm{g} / \mathrm{L}$ in the OW/OBNGR group, and $239 \pm 61 \mu \mathrm{g} / \mathrm{L}$ in the OW/OB-T2DM group. In both the diabetic and normal glucose regulation groups, the serum ATGL level was lower in the obese patients than in the non-obese patients, and the difference was statistically significant $(\mathrm{P}<0.05)$.

Pearson correlation analysis showed that the fasting serum ATGL level was negatively correlated with body fat content, BMI, WHR, TG, and HOMA-IR $(r=-0.271, r=-0.238, r=$ $-0.375, r=-0.313$, and $r=-0.164$, respectively; $\mathrm{P}<0.05)$. ATGL was also positively correlated with HDL-C $(r=0.497, \mathrm{P}<0.05)$, but was not significantly correlated with age, gender, fasting blood glucose, LDL-C, blood pressure, or HbA1c. The stepwise regression analysis was used with ATGL as the dependent variable (Y) and gender (X1), age (X2), BMI (X3), WHR (X4), body fat content (X5), systolic blood pressure (X6), diastolic blood pressure (X7), TC (X8), TG (X9), HDL-C (X10), glycemic index (X11), HOMA-IR (X12), and fasting insulin (X13) as the independent variables. The body fat content and WHR were retained in the regression equation: $\mathrm{Y}=10.018-0.275 \mathrm{X}_{4}-0.104 \mathrm{X}_{5}$.

\section{DISCUSSION}

ATGL was shown to be the rate-limiting enzyme of lipid hydrolysis, and some studies have shown that ATGL single nucleotide polymorphisms were significantly correlated with plasma free fatty acids, glucose levels, and type 2 diabetes (Schoenborn et al., 2006). Currently, few studies have been conducted investigating the relationship between obesity and diabetes and human serum ATGL content; therefore, this study aimed to explore this relationship. 
Langin et al. (2000) measured the subcutaneous adipose tissue in obese and normal human subjects, and found that the ATGL expression level in obese people was lower than that in normal people. ATGL expression decreased by 80 and $50 \%$, respectively, in obese and genetically induced ob/ob mice compared with that in the wild-type mice (Gibbons et al., 2000). It was found that the brummer gene ( $\mathrm{bmm})$, which encodes lipase in Drosophila, was similar to that encoding ATGL in humans, and that the lack of food or slow overexpression of the gene could deplete the body's storage of fat, and the lack of the gene may cause obesity (Mairal et al., 2006). Smirnova et al. (2006) also showed that overexpression of the ATGL gene in mammals could significantly reduce lipid droplets. In contrast, when RNA interference was used to silence the gene, the lipid droplets significantly increased. The results of the present study also showed that the serum ATGL levels were significantly lower in the overweight or obese group than those in the normal weight group. The serum ATGL levels showed a significantly negative correlation with anthropometric parameters such as BMI, body fat content, and WHR, and the stepwise multiple regression analysis revealed that the percentage of body fat and the WHR were independently associated factors affecting the serum ATGL level. The experimental results suggested that the serum ATGL level was closely related to the content of adipose tissue, suggesting that ATGL could be used as a useful indicator of obesity and its related diseases. The likely explanation for why BMI did not enter the regression equation is that because BMI is an indicator reflecting weight, it could not reflect body fat content and body fat distribution differences within the same BMI and different ages in men and women. However, the WHR could accurately reflect the subcutaneous and intra-abdominal fat distribution. In recent years, many cross-sectional epidemiological surveys and longitudinal studies have supported the hypothesis that the body fat-centered distribution could predict the incidence of type 2 diabetes. Some researchers have performed meta-analyses on the association of type 2 DM and the WHR, finding that the WHR was higher in type 2 DM patients than in the general population. In population-based diabetes risk screenings, the increase of the WHR was found to be more valuable than the increase of BMI (Zhang, 2006). Here, the ATGL was significantly correlated with the WHR, which reflected central obesity, suggesting that the serum ATGL level and the body fat distribution were closely related. ATGL plays an important role in lipid metabolism, obesity, and related diseases, and is therefore worthy of further exploration.

Some studies have found that the ATGL mRNA expression level in subcutaneous and visceral adipose tissue was associated with fasting insulin levels and insulin sensitivity (Berndt et al., 2008). The HOMA-IR index reflects the extent of insulin resistance in the body; greater HOMA-IR values reflect more severe insulin resistance. The results of our study showed that HOMA-IR and ATGL were negatively related, suggesting that ATGL may be involved in insulin resistance. Insulin is the main inhibitor of lipolysis, which has also drawn greater attention in ATGL-control studies. Kralisch et al. (2005) demonstrated that in the 3T3L1 fat cells of mice, insulin regulated the ATGL expression in a dose- and time-dependent manner. In the fat cells of induced diabetic rats, ATGL expression decreased due to insulin deficiency, but the ATGL expression significantly increased in the adipose tissue of rats once the insulin level recovered. The $100 \mathrm{nM}$ insulin treatment for $16 \mathrm{~h}$ could reduce ATGL mRNA expression by $49 \%$, and both the processing time and the dose could affect its expression (Gibbons et al., 2000). In the diabetic state, due to insulin resistance or insulin-secretory defects, the insulin signal would be lacking, and ATGL expression would enhance to eventually result in lipid metabolism disorders. This dyslipidemia can subsequently increase insulin resistance and create a vicious cycle. These findings showed that ATGL had an important connection 
with insulin resistance and insulin secretion defects.

Some studies have found that the serum ATGL levels have tight connections with glucose metabolism disorders in vivo (Wei et al., 2009; Xia and Weng, 2009). However, in this study, the correlation analysis showed that ATGL was not significantly correlated to HBA1c and fasting glucose levels. The reasons that ATGL was not correlated to blood glucose in this study were as follows: 1) the impact of the experimental conditions and small sample size, and 2) other cytokine secretion changes in the diabetes status may affect the level of ATGL. Therefore, the impact of blood glucose on ATGL needs further study.

We could only draw preliminary conclusions since research into the role of ATGL in metabolism is still relatively new, and because the sample size of this study was limited. Whether the serum ATGL level can indirectly reflect the ATGL activity in fat cells or the TG hydrolysis mode, and the detailed mechanisms of the hormonal regulation of ATGL and its protein expression and regulation activity currently remain unclear. ATGL may have a direct or indirect connection with obesity, insulin resistance, and/or type 2 diabetes, which provides a new way of thinking about obesity, and an entry point for the prevention of obesity-related diseases.

\section{ACKNOWLEDGMENTS}

Research supported in part by grants from the National Natural Science Foundation of China (\#81370965), the Natural Science Foundation of Jiangsu Province, China (\#BK2009208), the Medical Science and Technology Development Foundation and International Exchange Program of the Jiangsu Province Department of Health (\#H201247), the Science and Technology Commission of Zhenjiang City, Jiangsu Province (\#SH2013035, \#SH2012027), and the 13th Batch of Science and Technology Plan of Zhenjiang City in 2011 (\#SH2011052).

\section{REFERENCES}

Berndt J, Kralisch S, Klöting N, Ruschke K, et al. (2008). Adipose triglyceride lipase gene expression in human visceral obesity. Exp. Clin. Endocrinol. Diabetes 116: 203-210.

Deurenberg P, Weststrate JA and Seidell JC (1991). Body mass index as a measure of body fatness: age- and sex-specific prediction formulas. Br. J. Nutr. 65: 105-114.

Gibbons GF, Islam K and Pease RJ (2000). Mobilisation of triacylglycerol stores. Biochim. Biophys. Acta 1483: 37-57.

Kralisch S, Klein J, Lossner U, Bluher M, et al. (2005). Isoproterenol, TNFalpha, and insulin downregulate adipose triglyceride lipase in 3T3-L1 adipocytes. Mol. Cell Endocrinol. 240: 43-49.

Langin D, Lucas S and Lafontan M (2000). Millennium fat-cell lipolysis reveals unsuspected novel tracks. Horm. Metab. Res. 32: 443-452.

Mairal A, Langin D, Arner P and Hoffstedt J (2006). Human adipose triglyceride lipase (PNPLA2) is not regulated by obesity and exhibits low in vitro triglyceride hydrolase activity. Diabetologia 49: 1629-1636.

Schoenborn V, Heid IM, Vollmert C, Lingenhel A, et al. (2006). The ATGL gene is associated with free fatty acids, triglycerides, and type 2 diabetes. Diabetes 55: 1270-1275.

Smirnova E, Goldberg EB, Makarova KS, Lin L, et al. (2006). ATGL has a key role in lipid droplet/adiposome degradation in mammalian cells. EMBO Rep. 7: 106-113.

Wei L, Liu XH and Wang YL (2009). Serum fat triglyceride lipase level and its related factors in type 2 diabetes patients. Chin. Int. Med. 48: 407.

Xia X and Weng JP (2009). Adipose triglyceride lipase: a new kind of fat decomposition gene. Chin. J. Diabetes 10: 29-33.

Zhang XF (2006). The relationship between waist-to-hip ratio and type 2 diabetes, A meta-analysis. Changjiang Da Xue Xue Bao (Nat. Sci. Edn.) 3: 276-278.

Zimmermann R, Strauss JG, Haemmerle G, Schoiswohl G, et al. (2004). Fat mobilization in adipose tissue is promoted by adipose triglyceride lipase. Science 306: 1383-1386. 